\title{
rf losses in a high gradient cryogenic copper cavity
}

\author{
A. D. Cahill* and J. B. Rosenzweig \\ UCLA Los Angeles, California 90095, USA \\ V. A. Dolgashev, Z. Li, S. G. Tantawi, and S. Weathersby \\ SLAC Menlo Park, California 94025, USA
}

(Received 21 November 2017; published 29 June 2018)

\begin{abstract}
The development of high brightness electron sources can enable an increase in performance and reduction in size of extreme X-ray sources such as free electron lasers (FELs). A promising path to high brightness is through larger electric fields in radio-frequency (rf) photoinjectors. Recent experiments with $11.4 \mathrm{GHz}$ copper accelerating cavities at cryogenic temperatures have demonstrated $500 \mathrm{MV} / \mathrm{m}$ surface electric fields with low rf breakdown rates. However, when the surface electric fields are larger than $300 \mathrm{MV} / \mathrm{m}$, the measured cavity quality factor, $Q_{0}$, decreases during the input rf pulse by up to $30 \%$, recovering before the next rf pulse. In this paper, we present an experimental study of the rf losses, manifested as degradation of $Q_{0}$, in a copper cavity operated at cryogenic temperatures and high gradients. The experimental conditions range from temperatures of 10-77 K and rf pulse lengths of 100-800 ns, using surface electric fields up to $400 \mathrm{MV} / \mathrm{m}$. We developed a model for the change in $Q_{0}$ using measured field emission currents and rf signals. We find that the $Q_{0}$ degradation is consistent with the rf power being absorbed by strong field emission currents accelerated inside the cavity.
\end{abstract}

DOI: 10.1103/PhysRevAccelBeams.21.061301

Normal conducting copper cavities are commonly used when large accelerating gradients are required. For example, in high brightness electron sources for x-ray free electron lasers (FELs) such as LCLS and others [1-5] or in linear colliders for high energy particle physics, like the proposed NLC/GLC and CLIC [6,7]. Increasing accelerating gradients extends the potential reach of future energy frontier particle accelerators and is viewed as a way to mitigate their considerable cost. Furthermore, a gradient increase will enable more compact and thus accessible FELs [8]. However, sustained accelerating gradients in copper cavities are limited by vacuum rf breakdown [9-11]. With such compelling applications in mind, the physics of rf breakdown has been extensively studied, particularly the influence of rf electromagnetic fields and hardness of cavity material on breakdown probabilities [12-16].

One possible method to increase the electric fields sustained in copper cavities is to cool them to temperatures below $77 \mathrm{~K}$, where the rf surface resistance and coefficient of thermal expansion decrease, while the yield strength and thermal conductivity increase [17], all of which can

\footnotetext{
*acahill@physics.ucla.edu

Published by the American Physical Society under the terms of the Creative Commons Attribution 4.0 International license. Further distribution of this work must maintain attribution to the author(s) and the published article's title, journal citation, and DOI.
}

increase the limits of sustained surface fields. Of these material properties, the decrease in the rf surface resistance has been most intensively studied, which in cryogenic copper is well described by the theory of anomalous skin effect [18]. Measurements of the rf losses in cryogenicallycooled cavities are in good agreement with this theory over a wide range of frequencies [19-27]. To determine the $\mathrm{rf}$ surface resistance, these experiments have examined intrinsic quality factor, $Q_{0}$, of resonant cavities at very low fields utilizing sub-watt rf power.

However, there is little data on the $Q_{0}$ of copper cavities at temperatures below $100 \mathrm{~K}$ with high input rf power and surface electric fields greater than $75 \mathrm{MV} / \mathrm{m}$. rf surface resistance measurements obtained from an experiment performed at $9.3 \mathrm{GHz}$ and $77 \mathrm{~K}$, with $300 \mathrm{~kW}$ input peak power and associated surface electric fields up to $50 \mathrm{MV} / \mathrm{m}$ was consistent with low power measurements [25]. Another study conducted at $5.7 \mathrm{GHz}$ and $20 \mathrm{~K}$ with surface fields up to $65 \mathrm{MV} / \mathrm{m}$, also found high and low power $Q_{0}$ to be comparable [21]. However, an experiment at $3 \mathrm{GHz}$ and $77 \mathrm{~K}$ showed a decrease of $Q_{0}$ above $75 \mathrm{MV} / \mathrm{m}$ [27]. The authors report that the lowering of $Q_{0}$ is correlated with surface magnetic fields and may be caused by multipactor discharge [28].

In our previous experiments, we found that lowering the temperature of an X-band copper accelerating cavity allows it to sustain larger rf surface electric fields with decreased probability for vacuum rf breakdowns $[29,30]$. We observe 
that the $Q_{0}$ decreases for surface electric fields above $300 \mathrm{MV} / \mathrm{m}$. To understand both the improvement in high gradient performance and high field correlated-decrease in $Q_{0}$, we study the rf properties of copper at cryogenic temperatures excited by high power $11.4 \mathrm{GHz}$ rf waves that create up to $400 \mathrm{MV} / \mathrm{m}$ surface electric fields, in excess of those examined in the past.

To this end, we have developed a novel technique to measure the rf electric fields inside the cavity utilizing field emission currents, so called dark currents. Dark currents are created by electric fields at metal surfaces that promote bound electrons to a free propagating state. The magnitude of the dark currents is highly dependent on the surface electric field, according to the expression presented in [31], where the dependence of dark current versus field is given by the field enhancement factor $\beta$. For a given rf pulse, the $Q_{0}$ determines the surface fields, which in turn determines the strength of the dark currents. We use the sensitive dependence of dark currents on the electric fields to build an accurate model of the rf cavity performance.

The design of the cryogenic copper cavity for this experiment originated from an experimental program that has studied the physics of rf breakdowns in over 50 similar structures. The design, approach, and methods employed in these experiments can be found in [12,32-34]. The cavity has an iris radius of $2.75 \mathrm{~mm}$, and resonant frequency of $11.425 \mathrm{GHz}$ at $96 \mathrm{~K}$ [17]. The surface electromagnetic fields for the $\pi$ mode are shown in Fig. 1. The cavity does not have field probes, since they distort and amplify surface fields, degrading high power performance. To determine the electric fields, we instead use the measured input rf power to the cavity (forward power) and power reflected from the cavity both in tandem with signals from the current monitors that intercept the field emission currents.

The cavity is placed inside a cryostat and cooled by a pulse tube cryocooler, a Cryomech PT- 415 . The cold head of the cryocooling system is placed in thermal contact with the cavity [17], with the input waveguide connected to the exterior power source by a $\mathrm{TM}_{01}$ mode launcher [35]. For

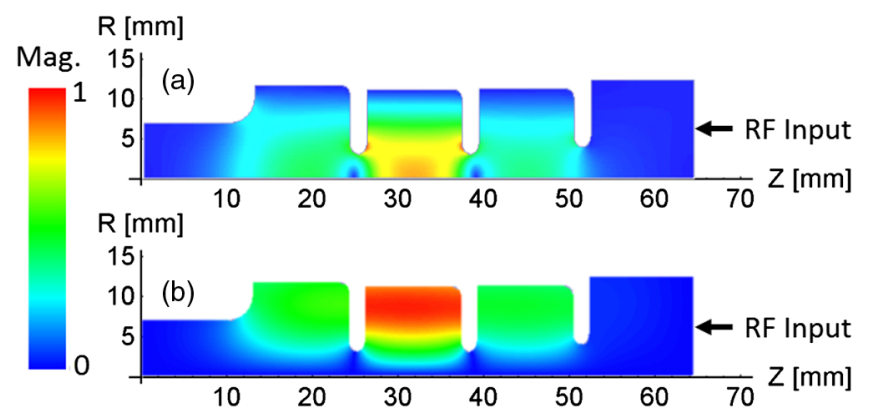

FIG. 1. Surface electric and magnetic fields for the cryogenic cavity. The fields are scaled to $2.5 \mathrm{MW}$ dissipated in the cavity, $T=45 \mathrm{~K}$ and $Q_{0}=30,263$. (a) shows the surface electric fields, with a maximum $400 \mathrm{MV} / \mathrm{m}$. (b) shows the surface magnetic fields, with a maximum $580 \mathrm{kA} / \mathrm{m}$. low power measurements, we used a vector network analyzer (VNA), a Keysight N5242A. With the VNA, we measure the dependence of the reflection coefficient on the $\mathrm{rf}$ frequency. Knowing this dependence, we calculate $Q_{0}$ and other rf parameters using a linear equivalent circuit [36]. For high power measurements, the rf source is a SLAC 50 MW XL-4 klystron that is pulsed with a repetition rate of $5-30 \mathrm{~Hz}$ and pulse length up to $1 \mu \mathrm{s}$. The forward and reflected signals are measured with a Keysight N1912A peak power meter and, through downmixing to $115 \mathrm{MHz}$, with readout through a fast digitizer. Two current monitors on both sides of the cavity intercept the field emitted electrons exiting the cavity and deliver signals to the digitizer via coaxial cables.

In numerous previous experiments with room temperature cavities, the rf and dark current signals have been well described by a linear equivalent circuit with a constant $Q_{0}$, determined from VNA measurements. We will refer to this equivalent circuit as the linear model. In this experiment, the linear model did not accurately predict the data, therefore we allowed the $Q_{0}$ and resonant angular frequency, $\omega_{0}$, to vary in time. This alternate model we refer to as the nonlinear model. When $Q_{0}$ and $\omega_{0}$ are timedependent, $\tilde{E}$, a variable proportional to the accelerating gradient, is governed by the following equation [37]:
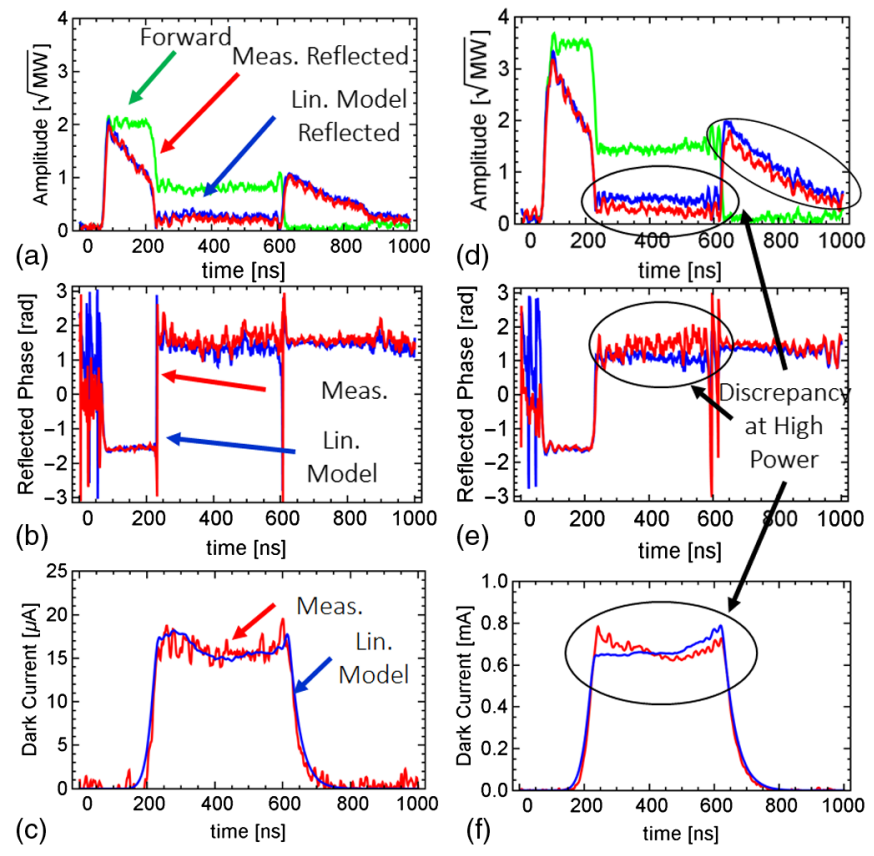

FIG. 2. Measurement (red) and linear model (blue) for two pulses with flat gradient section of $400 \mathrm{~ns}$ of low (left) and high (right) power at $45 \mathrm{~K}$ and $10 \mathrm{~Hz}$. The input rf pulse (green), begins at $50 \mathrm{~ns}$ and ends at $600 \mathrm{~ns}$. The model agrees with the measured signal for $Q_{0}=30,263$ at low power ((a)-(c)). For high power ((d)-(f)), the linear model does not match the measured rf and dark current signals. 


$$
\begin{aligned}
& \sqrt{\frac{8 P_{\text {in }}(t) \omega_{0}(t)^{3}}{\epsilon_{0} Q_{E}}} \\
& =\frac{d \tilde{E}(t)}{d t}\left(\frac{\omega_{0}(t)}{Q_{E}}+\Omega\left(\frac{1}{Q_{0}(t)}-2 i\right)\right) \\
& \quad+\tilde{E}(t)\left(\left(\omega_{0}(t)^{2}-\Omega^{2}\right)-i \Omega\left(\frac{\Omega}{Q_{0}(t)}+\frac{\omega_{0}(t)}{Q_{E}}\right)\right),
\end{aligned}
$$

where $\Omega$ is the driving frequency of the input $\mathrm{rf}, P_{\text {in }}$ is the input rf power, $Q_{E}$ is the external quality factor, $\epsilon_{0}$ is the vacuum permittivity, and $i=\sqrt{-1}$. We assumed $Q_{E}$ is constant in time and that $Q_{0} \gg 1$. Any variation of input phase is contained in the complex function $P_{\text {in }}(t)$, so $\Omega$ is also constant.

The time evolution of the fields inside the cavity depend both on the rf properties of the cavity and the shape of the input rf pulse. We shaped the klystron pulse to create constant electric fields for a portion of the pulse [33]. Figure 3 shows an example of such a pulse, with $t_{1}$ defined as the beginning of the rf pulse, $t_{2}$ the beginning of the flat part, and $t_{3}$ the end of the flat section. This pulse shape allows accurate determination of $Q_{0}(t)$, because small changes in $Q_{0}$ manifest as changes in the slope of the field during the flat part. The strong dependence of dark current on the electric fields allows us to detect these small changes in the slope. For our experimental conditions, we
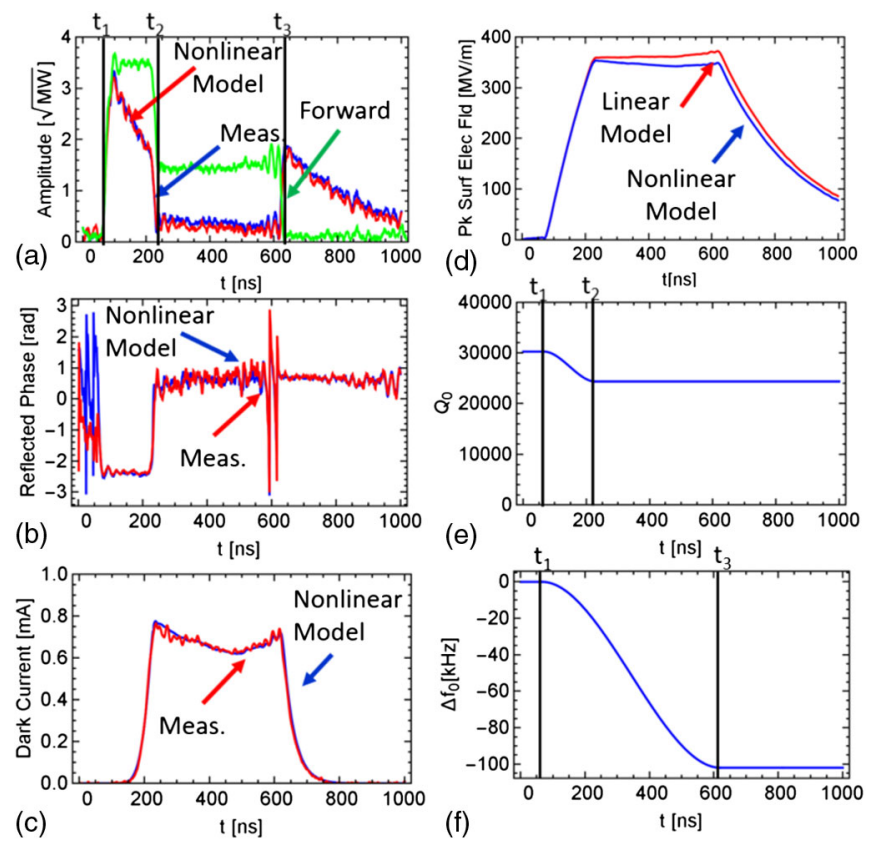

FIG. 3. Measured data (red) for $45 \mathrm{~K}$, also shown in Fig. 2(d)(f), and nonlinear model (blue) are plotted in (a)-(c). (d) shows the calculated gradient for linear and nonlinear models. (e) and (f) show the predicted change in $Q_{0}(t)$ and $f_{0}(t)=\omega_{0}(t) / 2 \pi$ respectively. Comparing the linear model in Fig. 2(d)-(f) and the nonlinear model in Fig. 3(a)-(c), the nonlinear model better predicts the data. found that using the flatness of the field gives a more accurate measurement of the rf losses than the other methods. It is more accurate to compare quantitatively the slope of the measured dark current, than measuring, for example, the time constants of the exponential signals.

A common way of measuring $Q_{0}$ is to measure the time constant of the exponential decay of the reflected rf power after the input pulse. We found that this technique was not effective in our case for two reasons. First, the data is distorted due to multiple reflections in the waveguide system between the cavity and klystron. Second, the difference in the time constants of the reflected power decay corresponding to the reduced $\mathrm{Q} 0$ value and the nominal Q0 value is small and buried in the noise. This is because at low temperatures the decay time constant, which is determined by Q loaded is dominated by the smaller external quality factor, QE. Knowing the facts above, we invented the more accurate method that uses dark current to determine the quality factor.

To fit our measurements to the nonlinear model using the dark currents, we first need to find the exact exponential dependence on surface electric fields, governed by the field enhancement $\beta$. We find $\beta$ for each pulse by fitting the exponential growth of the dark current to the calculated electric fields from $t_{1}$ to $t_{2}$. We use the linear model for this field calculation and assume $\beta$ is constant during the $\mathrm{rf}$ pulse. Next, with our nonlinear model we calculate the cavity fields and dark current using the obtained $\beta$. Then, we find $Q_{0}(t)$ by matching the measured to the calculated dark currents. We find $\omega_{0}(t)$ by fitting the rf phase of the reflected signal.

We only present results obtained at $45 \mathrm{~K}$, since the phenomenology of the $Q_{0}$ degradation does not vary for temperatures below $77 \mathrm{~K}$ [37]. At $45 \mathrm{~K}$, the $Q_{0}$ from VNA measurements was 30,263 [38], the external quality factor, $Q_{E}=15,361$, therefore the cavity is over-coupled with a $\beta=1.97$. For surface electric fields less than $300 \mathrm{MV} / \mathrm{m}$ the measured data was well predicted by the linear model, as shown in Fig. 2(a)-(c). For larger electric fields, there are clear deviations between the measured signals and those calculated with the linear model, as shown in Fig. 2(d)-(f). These discrepancies are large when compared to our previous experiments with room temperature cavities.

We tested many functional dependencies for $\omega_{0}(t)$ and $Q_{0}(t)$ and find that the model shown in Figs. 3(e) and 3(f) best describes the data. The initial value of $Q_{0}(t)$ is equal to its measured low power value and begins to decrease at time $t_{1}$ before becoming constant again at time $t_{2}$ [Fig 3(e)]. The $Q_{0}$ returns to its original value by the start of the next rf pulse; the enhanced losses are a reversible effect. However, in this experiment we cannot robustly determine exactly when the return to the initial value occurs. We find that the resonant frequency of the cavity changes over a longer time scale than $Q_{0}(t)$, where $\omega_{0}(t)$ begins to decrease at time $t_{1}$ and reaches a minimum value at time $t_{3}$ [Fig. 3(f)]. Again, 


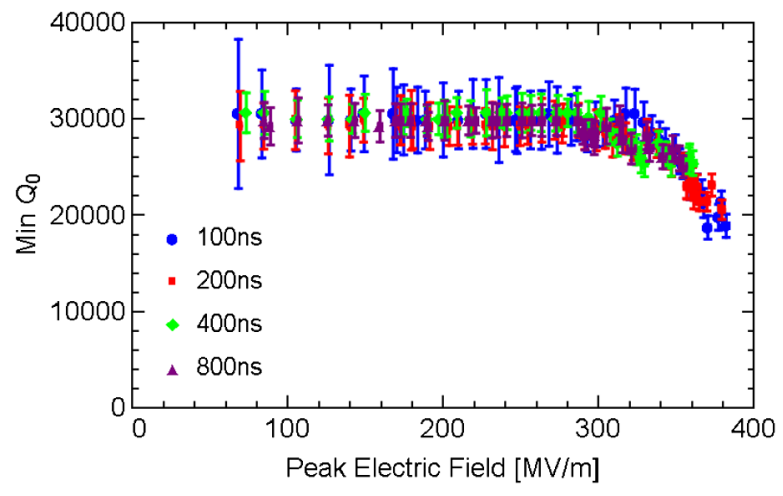

FIG. 4. $Q_{0}$ at the end of the rf pulse, when it reaches a minimum, versus calculated gradient for different rf pulse lengths. The plot shows no obvious dependence on pulse length, but a strong dependence on peak surface electric fields.

we cannot determine exactly when the $\omega_{0}$ recovers to its initial value, thus we leave $\omega_{0}$ at the minimum after $t_{3}$. In both cases, we have used a monotonic cubic spline between the starting and minimum value. We found that the measured reflected and dark current signals were well matched using the nonlinear model, as shown in Fig 3(a)-(c). In Fig. 3(d) we show the peak surface electric field calculated by both the linear and nonlinear models, where the difference in the maximum field is $6 \%$, even though the $Q_{0}$ drops by $20 \%$.

For fixed cavity temperatures, we have used this method to obtain $Q_{0}(t)$ and $\omega_{0}(t)$ for a range of input rf pulse lengths, $\mathrm{rf}$ repetition rates, and input rf powers. We find no dependence in the minimum $Q_{0}, Q_{0}\left(t_{2}\right)$, with variation of from 5 to $30 \mathrm{~Hz}$. This implies that average rf power does not affect the $Q_{0}$ degradation. We also find no clear evidence of $Q_{0}\left(t_{2}\right)$ dependence on the length of the input rf pulse, where the part of the pulse with the flat gradient varied from 100 to $800 \mathrm{~ns}$, as shown in Fig 4. This independence of $Q_{0}\left(t_{2}\right)$ on pulse length is inconsistent with the hypothesis that the surface rf resistance is increased by pulsed rf surface heating, since the maximum temperature change due to the pulsed surface heating, and thus any increase in rf surface resistance, increases with the pulse length [39].

Next, we introduce the quantity "missing rf power," which is the difference in dissipated rf power calculated by the linear and nonlinear models. We found that the missing power correlates with the magnitude of dark current measured in the current monitors, as shown in Fig 5. Therefore, we hypothesize that the degradation of $Q_{0}$ is caused by dark current beam loading, where field emitted electrons are accelerated by the electric field inside the cavity, and absorb power, which is then seen as missing from the rf system. The change in $\omega_{0}$ is also independent of repetition rate and $\mathrm{rf}$ pulse length. The beam loading could be responsible for the behavior of $\omega_{0}(t)$ as well as the change in $Q_{0}(t)$, by what is called the reactive component modifying the rf phase of the fields inside the cavity, which

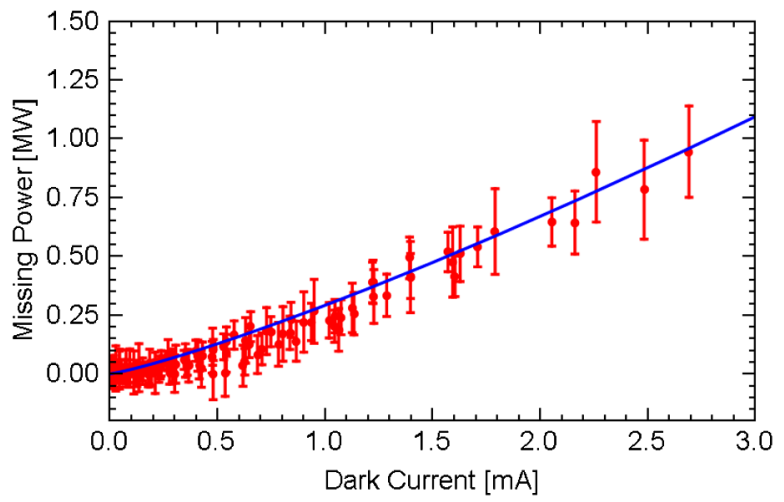

FIG. 5. Measured (red) and simulated (blue) missing power in the accelerating cavity versus the measured dark current magnitude for rf pulse lengths $100-800 \mathrm{~ns}$. The missing power is the difference between what we measured as the power lost to the cavity walls, and what would have been lost to the walls in the linear model. The simulation of power being lost to dark currents is consistent with the presented data.

would appear as a change of the resonant frequency [40]. The study of the physics of $\omega_{0}(t)$ in the current setup is difficult, future experiments will require more diagnostics.

To investigate the hypothesis of beam loading, we simulated field emitted electrons using a finite element electromagnetic and particle tracking code, ACE3P [41]. We used a simplified model of field emission, where electrons were emitted uniformly from the cavity walls using the same field emission model with $\beta=25$ and work function $\phi=4.2 \mathrm{eV}$ [31]. Particles lost in the walls or current monitors generated no secondary or elastically scattered electrons. As a result, the simulated electrons at the current monitors showed expected behavior, with the current exponentially dependent on the applied electric field and measured $\beta$ at the monitors close to the emission $\beta$. We understand some of the limitations of the model to be the following: the location of electron emitters is not known and thus uniform emission is assumed; secondary and elastically scattered electrons are ignored, which are considered in [42]; and ambient magnetic fields are ignored.

In these simulations, we calculated the average energy of the field emitted electrons when they were lost in the walls or current monitors. We calculate the missing power absorbed by the dark current by multiplying the measured dark current magnitude by the average electron energy. Since we do not know the ratio of total dark current that is intercepted by the current monitors we use this factor as a fitting parameter. The fitted value for this parameter is $0.0525 \%$. The results of the simulations together with measurements are shown in Fig. 5, where the simulation is consistent with the data.

We have used a cryogenically cooled $11.4 \mathrm{GHz}$ cavity to measure rf properties of copper from 10 to $77 \mathrm{~K}$, and from low power, with a VNA, to very high power, reaching peak surface electric fields up to $400 \mathrm{MV} / \mathrm{m}$. Measurements of 
the rf properties when surface electric fields were lower than $300 \mathrm{MV} / \mathrm{m}$, with both the klystron and VNA, were consistent with each other. For surface electric fields beyond $300 \mathrm{MV} / \mathrm{m}$, we observed a decrease in $Q_{0}$ and $\omega_{0}$. The decrease in $Q_{0}$ occurs simultaneously with the increase of electric fields inside the cavity. The $Q_{0}$ and $\omega_{0}$ return to their initial values before the next pulse for repetition rates of $5-30 \mathrm{~Hz}$.

We developed a model to determine the functions $Q_{0}(\mathrm{t})$ and $\omega_{0}(t)$ using the measured rf and current monitor signals. Since small changes in the rf surface electric fields lead to large changes in the field emission current, we can measure small variations in the surface electric field. In this way, we used the current monitors as field probes. The function $\omega_{0}(t)$ was determined from the phase of the reflected rf signal.

The observation that there is no obvious dependence of the $Q_{0}$ degradation on the rf pulse length in the range of 100$800 \mathrm{~ns}$ implies that rf pulsed surface heating is not a dominant effect on the $Q_{0}$ change. We further find no obvious dependence on the repetition rate of the input pulsed rf power in a range of $5-30 \mathrm{~Hz}$, indicating that average rf power has no significant effect either. Eliminating these above effects as the cause of $Q_{0}$ decrease, we find that the missing power lost in the cavity that manifests in the experiment as a reduction in $Q_{0}$, is correlated with the magnitude of the measured field emission currents.

We conclude that the dominant factor causing the degradation of $Q_{0}$ is $\mathrm{rf}$ power absorbed by accelerated field emission currents, or dark current beam loading, which is supported by simulations. Similar effects have been seen at high fields in room temperature cavities [43]. However, in our case of a cryogenic cavity the effect is more pronounced for two reasons. First, since the sustained surface electric fields are larger than typical room temperature experiments, more field emission currents are produced, greatly enhancing the beam loading. Second, since $Q_{0}$ is increased at cryogenic temperatures, the power lost due to beam loading is not overwhelmed by power lost to resistive losses. It is possible that other mechanisms of $\mathrm{rf}$ losses are also present, but due to the strong dependence of field emission current on surface fields, the effect of beam loading is observed to dominate in this experiment.

In the future we plan to verify how quickly the cavity parameters return to their initial values. We have proposed an rf pump-probe experiment, where the cavity is filled and emptied quickly while a second rf pulse will measure the time evolution of the rf parameters. As the field emitted electrons are limiting operating gradients of the accelerating cavity, we will explore methods of reducing the dark currents. Finally, we will continue this study at $2.8 \mathrm{GHz}$.

\section{ACKNOWLEDGMENTS}

This work was supported by U.S. NSF Grant No. PHY1549132, Center for Bright Beams and DOE/SU Contract No. DE-AC02-76-SF00515.
[1] P. Emma et al., First lasing and operation of an ångstromwavelength free-electron laser, Nat. Photonics 4, 641 (2010).

[2] M. Altarelli et al., European x-ray free electron laser, DESY Technical Report, 2006.

[3] R. Ganter et al., SwissFEL conceptual design report, Paul Scherrer Institute, Technical Report, 2012.

[4] S. Chen et al., SXFEL design report, Shanghai Institute of Applied Physics Technical Report, 2007.

[5] J. Han, in Proceedings of International Particle Accelerator Conference (IPAC'16), Busan, Korea, 2016, p. 6, ISBN: 978-3-95450-147-2.

[6] SLAC, Zeroth-order design report for the next linear collider, SLAC Technical Report, 1996.

[7] M. Aicheler, P. Burrows, M. Draper, T. Garvey, P. Lebrun, K. Peach, N. Phinney, H. Schmickler, D. Schulte, and N. Toge, A multi-TeV linear collider based on CLIC technology: CLIC conceptual design report, CERN Technical Report, 2012.

[8] J. B. Rosenzweig, A. Cahill, V. Dolgashev, C. Emma, A. Fukasawa, R. Li, C. Limborg, J. Maxson, P. Musumeci, A. Nause, R. Pakter, R. Pompili, R. Roussel, B. Spataro, and S. Tantawi, Next Generation High Brightness Electron Beams From Ultra-High Field Cryogenic Radiofrequency Photocathode Sources, arXiv:1603.01657.

[9] V. A. Dolgashev and S. G. Tantawi, in Proceedings of the 8th European Particle Accelerator Conference, Paris, 2002 (EPS-IGA and CERN, Geneva, 2002), p. 2139.

[10] J. W. Wang, High Energy Phys. Nucl. Phys. 30, 11 (2006).

[11] F. Wang, C. Adolphsen, and C. Nantista, Performance limiting effects in X-band accelerators, Phys. Rev. ST Accel. Beams 14, 010401 (2011).

[12] V. Dolgashev, S. Tantawi, Y. Higashi, and B. Spataro, Geometric dependence of radio-frequency breakdown in normal conducting accelerating structures, Appl. Phys. Lett. 97, 171501 (2010).

[13] S. Dobert et al., in Proceedings of the 21st Particle Accelerator Conference, Knoxville, TN, 2005 (IEEE, Piscataway, NJ, 2005), p. 372, ISSN: 1944-4680.

[14] C. Adolphsen, in Proceedings of the 2003 Particle Accelerator Conference, Portland, OR (IEEE, New York, 2003), Vol. 1, p. 668, ISSN: 1063-3928.

[15] V. A. Dolgashev, Progress on high-gradient structures, AIP Conf. Proc. 1507, 76 (2012).

[16] A. Grudiev, S. Calatroni, and W. Wuensch, New local field quantity describing the high gradient limit of accelerating structures, Phys. Rev. ST Accel. Beams 12, 102001 (2009).

[17] V. A. Dolgashev, J. R. Lewandowski, D. W. Martin, S. G. Tantawi, S. P. Weathersby, and A. D. Yeremian, in Proceedings of the 3rd International Particle Accelerator Conference, New Orleans, LA, 2012 (IEEE, Piscataway, NJ, 2012), Vol. C1205201, p. 3368.

[18] G. Reuter and E. Sondheimer, The theory of the anomalous skin effect in metals, Proc. R. Soc. A 195, 336 (1948).

[19] B. Pippard, The surface impedance of superconductors and normal metals at high frequencies II. The anomalous skin effect in normal metals, Proc. R. Soc. A 191, 385 (1947).

[20] T. Tanaka, T. Sakai, K. Nogami, K. Hayakawa, Y. Hayakawa, K. Nakao, K. Takatsuka, M. Fukuda, F. Satoh, T. Takatomi, N. Terunuma, J. Urakawa, and M. Yoshida, in 
Proceedings of International Particle Accelerator Conference (IPAC'17), Copenhagen, Denmark, 2017, p. 518, ISBN: 978-3-95450-182-3.

[21] A. Ino, S. Yamaguchi, T. Shintomi, T. Higo, Y. Funahashi, S. Matsumoto, S. Michizono, H. Endo, and K. Ueno, in Proceedings of 13th Annual Meeting of Part. Accel. Soc. of Japan, p. 863.

[22] F. Biquard and A. Septier, Amelioration de la conductivite superficielle du cuivre et de l'aluminium en hyperfrequences, par abaissement de temperature, Nucl. Instrum. Methods 44, 18 (1966).

[23] J. Benard, N. H. E. Minyawi, and N. T. Viet, Reduction of RF losses at $35 \mathrm{GHz}$ in high purity copper resonant cavities by cooling to cryogenic temperature, Rev. Phys. Appl. 13, 483 (1978).

[24] J. Guo, S. Tantawi, D. Martin, and C. Yoneda, Cryogenic rf material testing with a High-Q copper cavity, AIP Conf. Proc. 1299, 330 (2010).

[25] A. J. Saversky and I. S. Shchedrin, in Proceedings of the 15th Particle Accelerator Conference, PAC-1993, Washington, DC, 1993 (IEEE, New York, 1993), p. 1030.

[26] A. Cahill, A. Fukasawa, J. Rosenzweig, G. Bowden, V. Dolgashev, M. Franzi, S. Tantawi, P. Welander, C. Yoneda, J. Guo, and Y. Higashi, in Proceedings of International Particle Accelerator Conference (IPAC'16), Busan, Korea, 2016, p. 487, ISBN: 978-3-95450-147-2.

[27] A. H. McEuen, P. Lui, E. Tanabe, and V. Vaguine, Highpower operation of accelerator structures at liquid nitrogen temperature, IEEE Trans. Nucl. Sci. 32, 2972 (1985).

[28] R. A. Kishek, Y. Y. Lau, L. K. Ang, A. Valfells, and R. M. Gilgenbach, Multipactor discharge on metals and dielectrics: Historical review and recent theories, Phys. Plasmas 5, 2120 (1998).

[29] V. A. Dolgashev, A. D. Cahill, S. Weathersby, J. Lewandowski, A. Haase, C. Yoneda, and S. Tantawi, Presented at HG2015 (2015).

[30] A. Cahill, V. Dolgashev, J. Rosenzweig, S. Tantawi, and S. Weathersby, in Proceedings of International Particle Accelerator Conference (IPAC'17), Copenhagen, Denmark, 2017, p. 4395, ISBN: 978-3-95450-182-3.

[31] J. W. Wang and G. A. Loew, in Frontiers of accelerator technology. Proceedings, Joint US-CERN-Japan International School, Hayama and Tsukuba, Japan, 1996, p. 768.

[32] B. J. Munroe, A. M. Cook, M. A. Shapiro, R. J. Temkin, V. A. Dolgashev, L. L. Laurent, J. R. Lewandowski,
A. D. Yeremian, S. G. Tantawi, and R. A. Marsh, High power breakdown testing of a photonic band-gap accelerator structure with elliptical rods, Phys. Rev. ST Accel. Beams 16, 012005 (2013).

[33] R. A. Marsh, M. A. Shapiro, R. J. Temkin, V. A. Dolgashev, L. L. Laurent, J. R. Lewandowski, A. D. Yeremian, and S. G. Tantawi, X-band photonic band-gap accelerator structure breakdown experiment, Phys. Rev. ST Accel. Beams 14, 021301 (2011).

[34] V. Dolgashev, G. Gatti, Y. Higashi, O. Leonardi, J. Lewandowski, A. Marcelli, J. Rosenzweig, B. Spataro, S. Tantawi, and D. Yeremian, High power tests of an electroforming cavity operating at $11.424 \mathrm{GHz}$, J. Instrum. 11, P03010 (2016).

[35] C. Nantista, S. Tantawi, and V. Dolgashev, Low-field accelerator structure couplers and design techniques, Phys. Rev. ST Accel. Beams 7, 072001 (2004).

[36] T. Wangler, RF Linear Accelerators (Wiley, New York, 1998).

[37] A. D. Cahill, Ultra-high accelerating gradients in radiofrequency cryogenic copper structures, Ph.D. thesis, UCLA, 2017.

[38] A. Cahill, V. Dolgashev, M. Franzi, J. Rosenzweig, S. Tantawi, and S. Weathersby, in Proceedings of North American Particle Accelerator Conference (NAPAC'16), Chicago, IL, USA, 2016, p. 987, ISBN: 978-3-95450180-9.

[39] D. Pritzkau, RF pulsed heating, Ph.D. thesis, Stanford University, 2001.

[40] P. Wilson, High energy electron linacs: Applications to storage ring rf systems and linear colliders, SLAC Technical Report, 1991.

[41] K. Ko, A. Candel, L. Ge, A. Kabel, R. Lee, Z. Li, C. Ng, V. Rawat, G. Schussman, and L. Xiao, in Proceedings of the 25th International Linear Accelerator Conference, LINAC-2010, Tsukuba, Japan (KEK, Tsukuba, Japan, 2010), p. 1028.

[42] H. Xu, M. Shapiro, and R. Temkin, in Proceedings of North American Particle Accelerator Conference (NAPAC'16), Chicago, IL, USA, 2016, p. 971, ISBN: 978-395450-180-9.

[43] R. Bossart, J. Godot, H. Kugler, J. Madsen, A. Riche, and J. Strode, in Proceedings, 3rd European Particle Accelerator Conference, EPAC92: Berlin Germany, p. 1026. 